\title{
ON THE DUALIZATION OF SUBDIRECT EMBEDDINGS
}

\author{
By \\ F. SZÁSZ and R. WIEGANDT (Budapest) \\ To Professor G. ALEXITs on his 70th birthday
}

\section{§ 1. Introduction}

In the algebra there are several kinds of structure theorems which can be formulated without operations, using only homological tools. For instance, the wellknown fact that any universal algebra can be subdirectly embedded in a direct product of subdirectly irreducible algebras, can be formulated in a pure categorytheoretical manner. Now the question arises what its dual statement asserts. Our purpose is to give such a category which satisfies certain selfdual conditions, and making use of these, to prove structure theorems and their dual statements. The structure theorems themselves are, of course, well-known statements for algebraic stuctures. However, their duals yield some theorems of unusual type. About the possibility of the dualization there occurs some trouble. The most of the difficulties is at finding selfdual conditions being necessary to prove the theorems. So we must not make use of the condition 'every epimorphism is a normal one' which is fulfilled for groups, since its dual is false. Further the lattice of all congruencerelations of any universal algebra is a so-called compactly generated lattice. This fact plays a very important role in the proof of the theorem according to subdirect embeddings of universal algebras, nevertheless compactly generating is not a selfdual notion.

Applying the theorems proved for certain categories, we establish some particular theorems for rings, groups, modules, respectively.

In $\S 2$ we give a detailed enumeration of the usual notions and assertions of the theory of categories with respect to the importance of the dual notions and assertions, moreover, we form a system of selfdual conditions which will be satisfied by the category we are dealing with. $\S 3$ is devoted to the investigation of subdirect embeddings, subdirect irreducibility and to the dualization of those. In $\S 4$ we applicate the results developed before for rings, groups, module and abelian groups. Most of the applications are concerned with rings.

\section{$\S 2$. Preliminaries}

Let $\mathscr{C}$ be a category. The objects and maps of $\mathscr{C}$ will be denoted by small Latin and small Greek letters, respectively. By definition $\mathscr{C}$ satisfies the following conditions:

$\left(C_{1}\right)$ If $\alpha: a \rightarrow b$ and $\beta: b \rightarrow c$ are maps, then there is a uniquely defined map $\alpha \beta: a \rightarrow c$, which is called the product of the maps $\alpha$ and $\beta$;

$\left(\mathrm{C}_{2}\right)$ If $\alpha: a \rightarrow b, \beta: b \rightarrow c, \gamma: c \rightarrow d$ are maps, then $(\alpha \beta) \gamma=\alpha(\beta \gamma)$ holds.

$\left(C_{3}\right)$ For each object $a \in \mathscr{C}$ there is a map $\varepsilon_{a}: a \rightarrow a$, called the identity map of $a$ such that for each $\alpha: b \rightarrow a$ and $\beta: a \rightarrow c$ we have $\alpha \varepsilon_{a}=\alpha, \varepsilon_{a} \beta=\beta$. 
The dual category of the category $\mathscr{C}$, denoted by $\mathscr{C}^{*}$, consists of the same objects as $\mathscr{C}$, and $\alpha^{*}: b \rightarrow a$ is a map of $\mathscr{C}^{*}$ if and only if $\alpha: a \rightarrow b$ is a map of $\mathscr{C}$. Clearly $\left(\mathscr{C}^{*}\right)^{*}=\mathscr{C}$, and if a statement $P$ is true for category $\mathscr{C}$, then there is a dual statement $P^{*}$ which will be true for $\mathscr{C}^{*}$. In what follows we shall assume that the category $\mathscr{C}$ satisfies some additional assumptions. These requirements will be selfdual which means that both of $\mathscr{C}$ and $\mathscr{C}^{*}$ satisfy them. So any statement $P$ which can be proved for $\mathscr{C}$, will be true for $\mathscr{C}^{*}$ too. Hence statement $P^{*}$ is true for $\left(\mathscr{C}^{*}\right)^{*}=\mathscr{C}$.

Let $H(a, b)$ denote the class of all maps of $\mathscr{C}$ which map $a$ into $b$. An object $o \in \mathscr{C}$ is said to be a zero object if for any object $a$ of $\mathscr{C}$ both of the classes $H(a, o)$ and $H(o, a)$ contain only one map.

We assume that

$\left(\mathrm{C}_{4}\right) \mathscr{C}$ possesses zero objects.

Obviously also $\mathscr{C}^{*}$ contains zero objects. We shall say that $\mathscr{C}$ is a category with zero maps, if for any ordered pair of objects $a, b$ there is a map $\omega_{a b}: a \rightarrow b$ such that for any $\alpha: c \rightarrow a, \beta: b \rightarrow d$ we have $\alpha \omega_{a b}=\omega_{c b}$ and $\omega_{a b} \beta=\omega_{a d}$. If $\mathscr{C}$ possesses zero objects, then $\mathscr{C}$ is a category with zero maps (cf. KURoš-LivšITs- ŠULGEIFERTsalenko [8]). If there is no doubt between which objects the zero map operates, then that zero map will be shortly denoted by $\omega$.

A map $\alpha: a \rightarrow c$ will be called a monomorphism, if for any maps $\varrho: b \rightarrow a$, $\sigma: b \rightarrow a$ from $\varrho \alpha=\sigma \alpha$ it follows $\varrho=\sigma$.

A map $\alpha: c \rightarrow a$ will be called an epimorphism, if for any maps $\varrho: a \rightarrow b$, $\sigma: a \rightarrow b$ from $\alpha \varrho=\alpha \sigma$ it follows $\varrho=\sigma$.

The notion of epimorphism is dual to that of monomorphism in the sense that $\alpha$ is a monomorphism of $\mathscr{C}$ if and only if $\alpha^{*}$ is an epimorphism of $\mathscr{C}^{*}$.

The product of two monomorphism (if it exists) is again a monomorphism. If $\alpha \beta$ is a monomorphism, then $\alpha$ is also a monomorphism.
The product of two epimorphisms: (if it exists) is again an epimorphism. If $\beta \alpha$ is an epimorphism, then $\alpha$ is. also an epimorphism.

The statements are well-known (cf. KuRoš-LivŠITs-ŠULGEIFER-TSALENKo [8], or Mitchell [9]). Now we are going to give the definitions of some usual notions. together with their duals.

Let $\beta_{1}: b_{1} \rightarrow a$ and $\beta_{2}: b_{2} \rightarrow a$ be monomorphisms. We shall say that $\left(b_{2}, \beta_{2}\right) \leqq\left(b_{1}, \beta_{1}\right)$, if there exists a map $\varrho$ (which has to be a monomorphism) such that $\varrho \beta_{1}=\beta_{2}$. If both of $\left(b_{2}, \beta_{2}\right) \leqq\left(b_{1}, \beta_{1}\right)$ and $\left(b_{1}, \beta_{1}\right) \leqq$ $\leqq\left(b_{2}, \beta_{2}\right)$ hold then the pairs $\left(b_{1}, \beta_{1}\right)$ and $\left(b_{2}, \beta_{2}\right)$ are said to be equivalent. If $\left(b_{2}, \beta_{2}\right) \leqq\left(b_{1}, \beta_{1}\right)$ but they are not equivalent, then we shall write $\left(b_{2}, \beta_{2}\right)<\left(b_{1}, \beta_{1}\right)$. The equivalence classes of the relation thus defined will be called the subobjects of $a$. For
Let $\beta_{1}: a \rightarrow b_{1}$ and $\beta_{2}: a \rightarrow b_{2}$ be epimorphisms. We shall say that $\left(\beta_{2}, b_{2}\right) \leqq\left(\beta_{1}, b_{1}\right)$ if there exists a map. $\varrho$ (which has to be an epimorphism) such that $\beta_{1} \varrho=\beta_{2}$. If both of $\left(\beta_{2}, b_{2}\right) \leqq$ $\leqq\left(\beta_{1}, b_{1}\right)$ and $\left(\beta_{1}, b_{1}\right) \leqq\left(\beta_{2}, b_{2}\right)$ hold, then the pairs $\left(\beta_{1}, b_{1}\right)$ and $\left(\beta_{2}, b_{2}\right)$. are said to be equivalent. If $\left(\beta_{2}, b_{2}\right) \leqq$ $\leqq\left(\beta_{1}, b_{1}\right)$ but they are not equivalent, then we shall write $\left(\beta_{2}, b_{2}\right)<\left(\beta_{1}, b_{1}\right)$. The equivalence classes of the relation thus defined will be called the factorobjects of $a$. For convenience the: 
convenience the equivalence class represented by the pair $(b, \beta)$ will also be denoted by $(b, \beta)$.

A commutative diagram

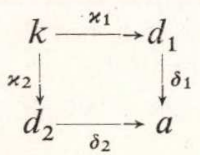

is called a pullback for $\delta_{1}$ and $\delta_{2}$, if for any object $c \in \mathscr{C}$ and commutative diagram

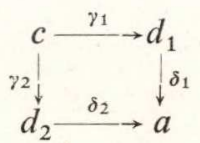

there exists a unique map $\gamma: c \rightarrow k$ such that the diagram

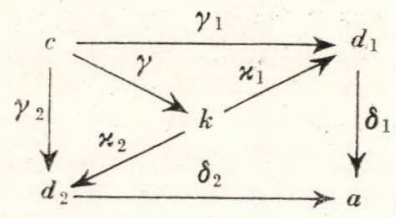

is again commutative.

A subobject $(k, x)$ of an object $a \in \mathscr{C}$ is said to be a kernel of the map $\alpha: a \rightarrow b$, if

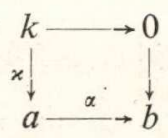

is a pullback diagram. Here the map $x$ has to be a monomorphism. Equivalently, the subobject $(k, x)$ is the kernel of $\alpha$ if (i) $x \alpha=\omega$; (ii) for each $\gamma: c \rightarrow a$ satisfying $\gamma \alpha=\omega$, there is a unique map $\gamma^{\prime}: c \rightarrow k$ such that $\gamma^{\prime} x=\gamma$. If $(k, x)$ is a kernel of $\alpha$, then we shall write $\operatorname{Ker} \alpha=(k, x)$, or only $\operatorname{Ker} \alpha=k$. The map $x$ is called a normal monomorphism and the subobject $(k, x)$ is a normal subobject or an ideal of $a$. equivalence class represented by the pair $(\beta, b)$ will also be denoted by $(\beta, b)$.

A commutative diagram

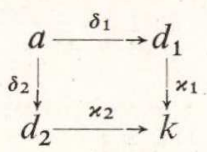

is called a pushout for $\delta_{1}$ and $\delta_{2}$, if for any object $c \in \mathscr{C}$ and commutative diagram

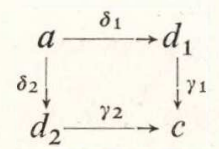

there exists a unique map $\gamma: k \rightarrow c$ such that

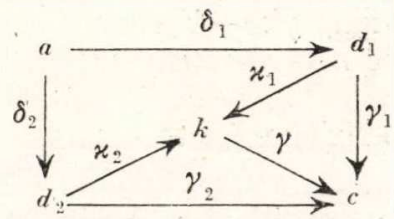

is again commutative.

A factorobject $(\varkappa, k)$ of an object $a \in \mathscr{C}$ is said to be a cokernel of the map $\alpha: b \rightarrow a$ if

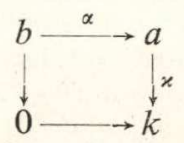

is a pusout diagram. Here the map $x$ has to be an epimorphism. Equivalently, the factorobject $(x, k)$ is the cokernel of $\alpha$ if (i) $\alpha x=\omega$; (ii) for each $\gamma: a \rightarrow c$ satisfying $\alpha \gamma=\omega$, there is a unique map $\gamma^{\prime}: k \rightarrow c$ such that $x \gamma^{\prime}=\gamma$. If $(x, k)$ is a cokernel of $\alpha$, then we shall write $\operatorname{Coker} \alpha=(\varkappa, k)$ or only Coker $\alpha=k$. The map $x$ is called a normal epimorphism and the factorobject $(x, k)$ is a normal factorobject of $a$. 
These definitions correspond to those of Mitchell [9] and SulińsKl [13]. In KuRoš-LivŠITS-ŠULGEIFER-TSALENKo [8] ideals and normal subobjects (and so their duals) are not the same notions, but under conditions supposed below they coincide.

In the category of groups every epimorphism is a normal one, but not every monomorphism is a normal one (i.e. not every subgroup is a normal subgroup). The product of two normal monomorphisms need not be a normal one. Moreover, if $\alpha$ is a monomorphism, then $\operatorname{Ker} \alpha=(o, \omega)$, but the converse statement does not hold.

If $\alpha \beta$ is a normal monomorphism and $\beta$ is a monomorphism, then $\alpha$ is a normal monomorphism. (Cf. [8] § 8.3). The dual statement also holds for normal epimorphisms.

We assume that

$\left(\mathrm{C}_{5}\right)$ Every map has a kernel and a cokernel.

Proposition 1. Ker Coker Ker $\alpha=\operatorname{Ker} \alpha$.

Proof. Let $\alpha: a \rightarrow b$ be a map, and put $\operatorname{Ker} \alpha=(k, x)$ and Coker $x=(\lambda, l)$. We have to prove $\operatorname{Ker} \lambda=(k, x)$. (i) Since $(\lambda, l)=$ Coker $x$, so by definition $x \lambda=\omega$ holds. (ii) Let $\gamma: c \rightarrow a$ be a map with $\gamma \lambda=\omega$. By definition of Coker $\chi$ there is a unique map $\gamma^{\prime}: c \rightarrow k$ such that $\gamma^{\prime} x=\gamma$. Thus from $\gamma \lambda=\omega$ we get the existence of a unique map $\gamma^{\prime}$ satisfying $\gamma^{\prime} x=\gamma$. Hence $\operatorname{Ker} \lambda=(k, x)$ is valid.

Dualizing we get

Proposition $1^{*}$. Coker Ker Coker $\alpha=\operatorname{Coker} \alpha$.

We suppose that

$\left(\mathrm{C}_{6}\right)$ The class of all subobjects and factorobjects of any object a is a set, and it forms a complete lattice $L_{a}$ and $L_{a}^{*}$ with respect to the relation $\leqq$ defined for subobjects and factorobjects, respectively.

$\left(\mathrm{C}_{7}\right)$ For each object $a \in \mathscr{C}$ the set of all normal subobjects and normal factorobjects, forms a complete sublattice of $L_{a}$ and $L_{a}^{*}$, respectively.

The intersection $\cap$ and union $U$ in the lattices $L_{a}$ and $L_{a}^{*}$ of the ideals and normal factorobjects of the objects $a$ can be defined in the following way.

The intersection $(k, x)$ of two ideals $\left(d_{1}, \delta_{1}\right),\left(d_{2}, \delta_{2}\right)$ is an ideal such that

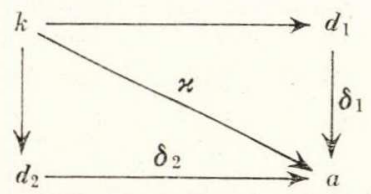

is a pullback diagram.
The intersection $(x, k)$ of two normal factorobjects $\left(\delta_{1}, d_{1}\right),\left(\delta_{2}, d_{2}\right)$ is a normal factorobject such that

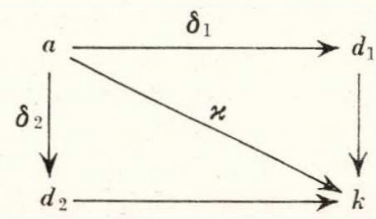

is a pushout diagram. 
The union $(l, \lambda)$ of two ideals $\left(d_{1}, \delta_{1}\right),\left(d_{2}, \delta_{2}\right)$ means an ideal for which

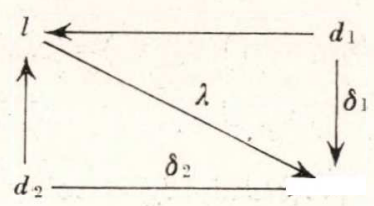

is a commutative diagram, and for any monomorphism $\gamma: c \rightarrow a$ and diagram

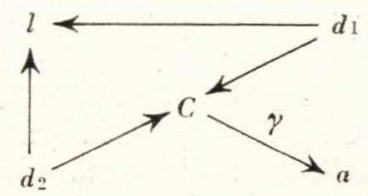

there is a monomorphism $\lambda^{\prime}: l \rightarrow c$ such that $\lambda^{\prime} \gamma=\lambda$, and the diagram becomes commutative.

The union of two normal factorobjects is defined in the dual way.

These definitions of the unions correspond to the relation $\leqq$ defined on $L_{a}$ and $L_{a}^{*}$, respectively. However, in Mitchell [9] the unions are defined in a somewhat different manner.

Proposition 2. The lattice $L_{a}$ of the ideals of an object a is dually isomorphic to the lattice $L_{a}^{*}$ of the normal factorobjects of $a$ in the following sense. Any ideal $(k, x)$ of $L_{a}$ is a kernel $\operatorname{Ker} \alpha$ of a map $\alpha$. The correspondence $\operatorname{Ker} \alpha \rightarrow$ Coker Ker $\alpha=$ $=(\lambda, l)$ is one-to-one, further the relation $\left(k_{1}, x_{1}\right) \leqq\left(k_{2}, x_{2}\right)$ holds if and only if $\left(\lambda_{1}, l_{1}\right) \geqq\left(\lambda_{2}, l_{2}\right)$ is valid for their cokernels in $L_{a}^{*}$.

Proof. Proposition 1 implies that $\operatorname{Ker} \alpha \rightarrow$ Coker $\operatorname{Ker} \alpha$ is a one-to-one correspondence.

Assume $\left(k_{1}, x_{1}\right) \leqq\left(k_{2}, x_{2}\right) \in L_{a}$, and put Coker $x_{i}=\left(\lambda_{i}, l_{i}\right), \quad i=1,2$. By definition

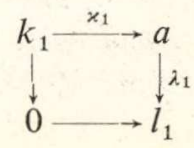

is a pushout diagram. Since $\left(k_{1}, \varkappa_{1}\right) \leqq\left(k_{2}, \varkappa_{2}\right)$, so there is a map $\varkappa^{\prime}: k_{1} \rightarrow k_{2}$ such that $x^{\prime} x_{2}=x_{1}$. Thus

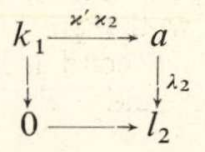

is a commutative diagram, and since (1) is a pushout, therefore there is a map $\lambda^{\prime}: l_{1} \rightarrow l_{2}$ such that $\lambda_{1} \lambda^{\prime}=\lambda_{2}$. This means $\left(\lambda_{1}, l_{1}\right) \geqq\left(\lambda_{2}, l_{2}\right)$. 
Dualizing, $\quad\left(\lambda_{1}, l_{1}\right) \geqq\left(\lambda_{2}, l_{2}\right)$ implies $\left(k_{1}, x_{1}\right) \leqq\left(k_{2}, x_{2}\right)$. Thus proposition 2 is proved. One can formulate this statement as follows:

are valid if and only if

$$
\begin{aligned}
& \left(k_{1}, x_{1}\right) \cap\left(k_{2}, x_{2}\right)=(c, \gamma), \\
& \left(k_{1}, x_{2}\right) \cup\left(k_{2}, x_{2}\right)=(d, \delta)
\end{aligned}
$$

$$
\begin{aligned}
& \left(\lambda_{1}, l_{1}\right) \cup\left(\lambda_{2}, l_{2}\right)=\text { Coker } \gamma, \\
& \left(\lambda_{1}, l_{1}\right) \cap\left(\lambda_{2}, l_{2}\right)=\text { Coker } \delta
\end{aligned}
$$

are valid.

Let $\alpha: a \rightarrow b$ be a map. If $\mu: a \rightarrow m$ is an epimorphism and $v: m \rightarrow b$ a monomorphism with $\mu \nu=\alpha$, then the subobjects $(m, v)$ of $b$ will be called an image of $\alpha$ (with the epimorphism $\mu$ ), $(m, v)$ is said to be a normal image, if $\mu$ is a normal epimorphism.

Let $(k, x)$ be a subobject of the object $a$ and let $\alpha: a \rightarrow b$ be an epimorphism. If $(m, v)$ is an image of $x \alpha$, then $(m, v)$ will be called an image of $(k, x)$ by the epimorphism $\alpha$.
Let $\alpha: b \rightarrow a$ be a map. If $\mu: m \rightarrow a$ is a monomorphism and $v: b \rightarrow m$ is an epimorphism with $v \mu=\alpha$, then the factorobject $(v, m)$ of $b$ will be called a coimage of $\alpha$ (with the monomorphism $\mu),(v, m)$ is said to be a normal coimage, if $\mu$ is a normal monomorphism.

Let $(x, k)$ be a factorobject of the object $a$ and let $\alpha: b \rightarrow a$ be a monomorphism. If $(v, m)$ is a coimage of $\alpha k$, then $(v, m)$ will be called a coimage of $(x, k)$ by the monomorphism $\alpha$.

A normal image (and normal coimage) is uniquely determined, but image (and coimage) is not (cf. KUROš-LivŠITS-ŠULGEIFER-TSALENKo [8]). If $(m, v)$ is an image of $b$ such that for every image $\left(m^{\prime}, v^{\prime}\right)$, of $b(m, v) \leqq\left(m^{\prime}, v^{\prime}\right)$, then $(m, v)$ will be denoted by $\operatorname{Im} \alpha$. Coim $\alpha$ will denote the dual notion.

In the category of groups or rings, for any map $\alpha$ both of $\operatorname{Im} \alpha$ and $\operatorname{Coim} \alpha$ does exist, moreover, $\operatorname{Im} \alpha$ is always a normal image, but Coim $\alpha$ need not be a normal coimage.

Let us assume that

$\left(\mathrm{C}_{8}\right)$ For any map $\alpha$ there exist $\operatorname{Im} \alpha$ and $\operatorname{Coim} \alpha$ (they need not be normal).

$\left(\mathrm{C}_{9}\right)$ An im ageof an ideal by a normal epimorphism is always a normal ideal, and a coimage of a normal factorobject by a normal monomorphism is always a normal factorobject.

Obviously all axioms $\left(\mathrm{C}_{1}\right)-\left(\mathrm{C}_{8}\right)$ are satisfied in the category of groups or rings. This category satisfies clearly the first condition of axiom $\left(\mathrm{C}_{9}\right)$. Also the second condition is fulfilled. Consider the coimage $(v, M)$ of a normal factorobject $(\varkappa, K)$ by a monomorphism $\alpha: B \rightarrow A$. Now the group (or ring) $K$ is a factorgrcup $A / C$ and $B$ is a subgroup of $A$. By the Second Isomorphism Theorem $B / B \cap C$ is isomorphic to a subgroup $B^{\prime} / C$ of $A / C$, and if $B$ is a normal subgroup of $A$, then $B^{\prime} / C$ is also a normal one of $A / C$.

PROPOSITION 3. If the map $\alpha$ has a normal image and $\operatorname{Ker} \alpha=(o, \omega)$, then $\alpha$ is a monomorphism. 

$\S 10,6$.

This statement is proved in KURO ̌̌-LIVŠITs-ŠULGEIFER-TSALENKo [8]

Let $a_{i}(i \in I)$ be a family of objects of the category $\mathscr{C}$.

An object $g$ is said to be a direct product of the objects $a_{i}(i \in I)$, if there are maps $\pi_{i}: g \rightarrow a_{i}(i \in I)$ (called the projections of $g$ onto $a_{i}$ ) such that for each object $h \in \mathscr{C}$ and for any system of maps $\alpha_{i}: h \rightarrow \alpha_{i}(i \in I)$, there is a unique map (called the canonical map) $\gamma: h \rightarrow g$ such that $\gamma \pi_{i}=\alpha_{i}$ for all $i \in I . g$ will be denoted by $g=$ $=\prod_{i \in I} a_{i}\left(\pi_{i}\right)$.
An object $f$ is said to be a free product of the objects $a_{i}(i \in I)$ if there are maps $\varrho_{i}: a_{i} \rightarrow f(i \in I)$ (called the injections of $a_{i}$ into $f$ ) such that for each object $h \in \mathscr{C}$ and for any system of maps $\alpha_{i}: a_{i} \rightarrow h \quad(i \in I)$ there is a unique map (called the canonical map) $\gamma: f \rightarrow h$ such that $\varrho_{i} \gamma=\alpha_{i}$ for all $i \in I$. $f$ will be denoted by $f=\sum_{i \in I} a_{i}\left(\varrho_{i}\right)$.

Assume that

$\left(\mathrm{C}_{10}\right)$ Every family of objects has a direct product and a free product.

Axiom $\left(\mathrm{C}_{4}\right)$ implies that all the projections $\pi_{i}$ (injections $\varrho_{i}$ ) of a direct product $g=\prod_{i \in I} a_{i}\left(\pi_{i}\right)$ (free product $f=\sum_{i \in I} a_{i}\left(\varrho_{i}\right)$ ) are epimorphism (monomorphisms). Moreover, to every projection $\pi_{i}$ there is a normal monomorphism $\sigma_{i}: a_{i} \rightarrow g$ such that $\sigma_{i} \pi_{i}=\varepsilon_{a_{i}}$ and $\sigma_{i} \pi_{j}=\omega(i \neq j)$ hold, and so $\left(a_{i}, \sigma_{i}\right)$ is an ideal of $g$ (dually: to every injection $\varrho_{i}$ there is a normal epimorphism $\tau_{i}: f \rightarrow a_{i}$ satisfying $\varrho_{i} \tau_{i}=\varepsilon_{a_{i}}$, $\left.\varrho_{j} \tau_{i}=\omega(i \neq j)\right)$. These facts are proved in [8].

Profosition 4. Let $\left(k_{i}, x_{i}\right)(i \in I)$ be a family of ideals of an object $a \in \mathscr{C}$, and let $\alpha_{i}: a \rightarrow a_{i}$ be epimorphisms with $\operatorname{Ker} \alpha_{i}=\left(k_{i}, x_{i}\right)(i \in I)$. Consider the direct product $g=\prod_{i \in I} a_{i}\left(\pi_{i}\right)$, and the canonical map $\gamma: a \rightarrow g\left(\gamma \pi_{i}=\alpha_{i}, i \in I\right)$. Then Ker $\gamma=\bigcap_{i \in I}\left(k_{i}, x_{i}\right)$ is valid.

For the proof we refer to SuLIŃski [13], Proposition 2. 1. We omit to formulate the dual statement.

An object $a \in \mathscr{C}$ is said to be $s u b$ directly embedded in the direct product $g=\prod_{i \in \boldsymbol{I}} a_{i}\left(\pi_{i}\right)$ if there exists a monomorphism $\gamma: a \rightarrow g$ such that all maps $\alpha_{i}=\gamma \pi_{i}: a \rightarrow a_{i}(i \in I)$ are normal epimorphisms (cf. [13]).
An object $a \in \mathscr{C}$ is said to be a transfree image of the free product $f=\sum_{i \in I} a_{i}\left(\varrho_{i}\right)$, if there exists an epimorphisms $\sigma: f \rightarrow a$ such that all maps $\beta_{i}=\varrho_{i} \sigma_{i}: a_{i} \rightarrow a(i \in I)$ are normal monomorphisms.

Let us remark that according to this definition generally $g$ can not be embedded subdirectly in itself, for the projections $\pi_{i}$ need not be normal epimorphisms. The dual consideration holds for transfree images. 
Proposition 5. An object $a \in \mathscr{C}$ can be subdirectly embedded in the direct product $g=\prod_{i \in I} a_{i}\left(\pi_{i}\right)$ if and only if there is a family of ideals $\left(k_{i}, x_{i}\right)(i \in I)$ of a such that each of them is the kernel of the normal epimorphism $\alpha_{i}: a \rightarrow a_{i}(i \in I)$ and $\bigcap_{i \in I}\left(k_{i}, x_{i}\right)=$ $=(o, \omega)$ holds .

Dualizing we obtain

Proposition $5^{*}$. An object a $\in \mathscr{C}$ is a transfree image of the free product $f=\sum_{i \in I} a_{i}\left(\varrho_{i}\right)$ if and only if there is a family of normal factorobjects $\left(\lambda_{i}, l_{i}\right)(i \in I)$, of a such that each of them is the cokernel of the normal monomorphism $\beta_{i}: a_{i} \rightarrow a(i \in I)$ and $\bigcap_{i \in I}\left(\lambda_{i}, l_{i}\right)=(\omega, o)$ holds.

The statement of Proposition 5 is proved in SulińsKI [13] (Theorem 2, 3), assumed that every epimorphism is a normal one. Thus we give a modificated proof of this assertion.

Let $a$ be subdirectly embedded in $g$ by a monomorphism $\gamma: a \rightarrow g$. Now every $\alpha_{i}=\gamma \pi_{i}(i \in I)$ is a normal epimorphism. If $\left(k_{i}, x_{i}\right)=\operatorname{Ker} \alpha_{i}$, then by Proposition 4 we get $\operatorname{Ker} \gamma=\bigcap_{i \in I}\left(k_{i}, x_{i}\right)$. Since $\gamma$ is a monomorphism, therefore $\bigcap_{i \in I}\left(k_{i} ; x_{i}\right)=$ $=(o, \omega)$ is valid.

Conversely, let $\left(k_{i}, x_{i}\right)$ be a family of ideals of $a$ such that $\left(k_{i}, x_{i}\right)=\operatorname{Ker} \alpha_{i}$ where $\alpha_{i}: a \rightarrow a_{i}$ are normal epimorphisms and $\bigcap_{i \in I}\left(k_{i}, x_{i}\right)=(o, \omega)$ holds. Then there is a map $\gamma: a \rightarrow g$ such that $\gamma \pi_{i}=\alpha_{i}$ for $i \in I$. Applying Proposition 4, we get Ker $\gamma=$ $=\bigcap_{i \in \boldsymbol{I}}\left(k_{i}, x_{i}\right)=(o, \omega)$. By Proposition 2 we obtain $\bigcup_{i \in \boldsymbol{I}}\left(\alpha_{i}, a_{i}\right)=\operatorname{Coker} \omega=\left(\varepsilon_{a}, a\right)$. Consider $\operatorname{Im} \gamma=(m, v)$ with the epimorphism $\mu$ (i.e. $v$ is a monomorphism and $\gamma=\mu v)$. Since $\alpha_{i}=\mu v \pi_{i}$ and $\alpha_{i}(i \in I)$ is an epimorphism, so $v \pi_{i}$ is also an epimorphism. Thus $(\mu, m) \geqq\left(\alpha_{i}, a_{i}\right)$ holds for every $i \in I$. Therefore we have $(\mu, m) \geqq \bigcup_{i \in I}\left(\alpha_{i}, a_{i}\right)=$ $=\left(\varepsilon_{a}, a\right)$. So $(\mu, m)$ is equivalent to $\left(\varepsilon_{a}, a\right)$, and $\mu$ is a normal epimorphism. Therefore Proposition 3 implies that $\gamma$ is a monomorphism, and Proposition 5 is proved.

An object $a \in \mathscr{C}$ is said to be subdirectly irreducible, if the intersection all of its non-zero ideals is a non-zero ideal.

An object $a \in \mathscr{C}$ is said to be transfreely irreducible, if the intersection all of its non-zero normal factorobjects is a non-zero normal factorobject.

According to Proposition 2, an object $a \in \mathscr{C}$ is transfreely irreducible if and only if the join of all its ideals $\neq\left(a, \varepsilon_{a}\right)$ differs from $\left(a, \varepsilon_{a}\right)$.

Finally, let us mention that the categories of all rings and groups, respectively, and their dual categories fulfill axioms $\left(C_{1}\right)-\left(C_{10}\right)$. 


\section{$\S$ 3. Subdirect embeddings and transfree images}

It is well-known that any universal algebra can be subdirectly embedded in a direct product of subdirectly irreducible universal algebras (G. BIRKHOFF [4]). In the proof there is making use of the fact that the lattice of congruence-relations of any universal algebra is compactly generated.

Let $L$ be a complete lattice. An element $k \in L$ is said to be a compact element, if $k \leqq \bigcup_{i \in I} l_{i}$ implies $k \leqq \bigcup_{j \in J} l_{j}$ for some finite $J \subseteq I$. The lattice is called compactly generated, if $L$ is complete and every element of $L$ is a union of (an infinite number of) compact elements.

In his paper [13] SULIŃSKI asked whether every object of a category satisfying somewhat stronger conditions than $\left(\mathrm{C}_{1}\right)-\left(\mathrm{C}_{10}\right)$, can be subdirectly embedded in a direct product of subdirectly irreducible objects. Concerning this problem for a category $\mathscr{C}$ satisfying axioms $\left(\mathrm{C}_{1}\right)-\left(\mathrm{C}_{10}\right)$ we present

THEOREM 1. If the lattice $L_{a}$ of all ideals of an object $a \in \mathscr{C}$ is compactly generated, then a can be subdirectly embedded in a direct product $g=\prod_{i \in I} a_{i}\left(\pi_{i}\right)$ by a monomorphism $\gamma$ such a way that every $\gamma \pi_{i}=\alpha_{i}(i \in I)$ is a normal epimorphism. A normal factorobject $a_{i}$ of this decomposition is subdirectly irreducible if and only if the following condition holds:

(I) For any normal factorobject $(\chi, m) \neq\left(\varepsilon_{a_{i}}, a_{i}\right)$ of $a_{i}$ (which is clearly a factorobject $\left(\chi_{1}, m\right)$ of a) there exists a normal factorobject $(\delta, d)$ of a such that $\left(\alpha_{i}, a_{i}\right)>$ $>(\delta, d) \geqq\left(\chi_{1}, m\right)$.

REMARK. Condition (I) seems to be complicated, but in the category of groups and rings, respectively, (I) is trivially fulfilled, for $\operatorname{Im} \alpha$ is always a normal image. However, its dual will be a rather natural condition in Theorem $1^{*}$. By Proposition 2 condition (I) means that for any ideal $\left(m^{\prime}, \chi^{\prime}\right) \neq(o, \omega)$ of $a_{i}$, there exists an ideal $\left(d^{\prime}, \delta^{\prime}\right) \geqq \operatorname{Ker} \alpha_{i}$ of $a$ such that for its image $\left(n^{\prime}, v^{\prime}\right)$ by $\alpha_{i}$ we have $(o, \omega) \neq$ $\neq\left(n^{\prime}, v^{\prime}\right) \leqq\left(n^{\prime}, \chi^{\prime}\right)$.

Proof. Let $(k, x) \neq(o, \omega)$ be a compact element of the lattice $L_{a}$ of all ideals of an object $a \in \mathscr{C}$. Consider the set $S_{k}=\left\{\left(l_{j}, \lambda_{j}\right)\right\}_{j \in J}$ of all ideals of $a$ for which $(k, x) \cap\left(l_{j}, \lambda_{j}\right)<(k, x)$. Let $\left(l_{1}, \lambda_{1}\right)<\left(l_{2}, \lambda_{2}\right)<\cdots<\left(l_{n}, \lambda_{n}\right)<\cdots$ an ascending chain of ideals from $S_{k}$, and denote $\bigcup_{n}\left(l_{n}, \lambda_{n}\right)$ by $\left(l_{0}, \lambda_{0}\right)$. We will show that $(k, x) \cap$ $\cap\left(l_{0}, \lambda_{0}\right)<(k, x)$. Otherwise it would be $(k, x)=\left(l_{0}, \lambda_{0}\right)$ and since $(k, x)$ is a compact element of $L_{a}$, so for an index $n_{0}$ a relation $(k, x) \leqq\left(l_{n_{0}}, \lambda_{n_{0}}\right)$ would hold in contradiction to the assumption. Making use of Zorn's lemma we obtain the existence of a maximal element $(l, \bar{\lambda})$ of $S_{k}$.

To any compact element $\left(k_{i}, x_{i}\right)(i \in I)$ of $L_{a}$, consider a maximal element $\left(\bar{l}_{i}, \bar{\lambda}_{i}\right)$ of $S_{k_{i}}$. Now we shall show $\bigcap_{i \in I}\left(\bar{l}_{i}, \bar{\lambda}_{i}\right)=(o, \omega)$. On the contrary, suppose $\left(l^{\prime}, \lambda^{\prime}\right)=$ $=\bigcap_{i \in I}\left(\bar{l}_{i}, \bar{\lambda}_{i}\right) \neq(o, \omega)$. Since $L_{a}$ is compactly generated, so $\left(l^{\prime}, \lambda^{\prime}\right)$ is a union $\bigcup_{t \in T}\left(k_{t}, x_{t}\right)$ of compact elements $\left(k_{t}, x_{t}\right) \neq(o, \omega)$. The maximal elements $\left(\bar{l}_{t}, \bar{\lambda}_{t}\right)$ of $S_{k_{t}}$ belonging to $\left(k_{t}, x_{t}\right)$ occur in the intersection representation of $\left(l^{\prime}, \lambda^{\prime}\right)$. Thus we get $\left(k_{t}, x_{t}\right) \leqq$ 
$\leqq\left(l^{\prime}, \lambda^{\prime}\right) \leqq\left(\bar{l}_{t}, \bar{\lambda}_{t}\right)$ which implies $\left(k_{t}, \varkappa_{t}\right) \cap\left(\bar{l}_{t}, \bar{\lambda}_{t}\right)=\left(k_{t}, x_{t}\right)$ contradicting the choice of $\left(\bar{l}_{t}, \bar{\lambda}_{t}\right)$.

Now, consider $\left(\alpha_{i}, a_{i}\right)=$ Coker $\bar{\lambda}_{i}$. Since $\left(\bar{l}_{i}, \bar{\lambda}_{i}\right)$ is an ideal, therefore by Proposition 1 we have $\operatorname{Ker} \alpha_{i}=\left(\bar{l}_{i}, \bar{\lambda}_{i}\right)=\operatorname{Ker} \operatorname{Coker} \bar{\lambda}_{i}$, further $\alpha_{i}$ is a normal epimorphism for all $i \in I$. Hence by virtue of Proposition $5 a$ can be subdirectly embedded in a direct product $g=\prod_{i \in I} a_{i}\left(\pi_{i}\right)$ by a monomorphism $\gamma: a \rightarrow g$ such that every map $\gamma \pi_{i}=\alpha_{i}$ is a normal epimorphism.

Finally, assume (I) for an object $a_{i}$. Since $\left(\alpha_{i}, a_{i}\right)>(\delta, d)$ so by Proposition 2 for their kernels we obtain $\left(\bar{l}_{i}, \bar{\lambda}_{i}\right)=\operatorname{Ker} \alpha_{i}<\operatorname{Ker} \delta=\left(d^{\prime}, \delta^{\prime}\right)$. By the choice of $\left(\bar{l}_{i}, \bar{\lambda}_{i}\right)$ it follows $\left(k_{i}, x_{i}\right) \leqq\left(d^{\prime}, \delta^{\prime}\right)$ where $\left(k_{i}, x_{i}\right)$ denotes the compact element of $L_{a}$ belonging to $\left(\bar{l}_{i}, \bar{\lambda}_{i}\right)$. Thus for the intersection $\left(d_{0}^{\prime}, \delta_{0}^{\prime}\right)$ of all ideals $\left(d^{\prime}, \delta^{\prime}\right)>\left(\bar{l}_{i}, \bar{\lambda}_{i}\right)$ we have $\left(k_{i}, x_{i}\right) \leqq\left(d_{0}^{\prime}, \delta_{0}^{\prime}\right)$. Again, by Proposition 2 for Coker $x_{i}=$ $=\left(\kappa_{0}, k_{0}\right)$ and Coker $\delta_{0}^{\prime}=\left(\delta_{0}, d_{0}\right)$ we get $\left(\kappa_{0}, k_{0}\right) \geqq\left(\delta_{0}, d_{0}\right)$ and $\left(\alpha_{i}, a_{i}\right) \geqq\left(\delta_{0}, d_{0}\right)$. Hereby

$$
\left(\varkappa_{0}, k_{0}\right) \cup\left(\alpha_{i}, a_{i}\right)>\left(\varkappa_{0}, k_{0}\right) \geqq\left(\delta_{0}, d_{0}\right)
$$

and so $\left(\alpha_{i}, a_{i}\right)>\left(\delta_{0}, d_{0}\right)$ follows. On the other hand for any normal factorobject $(\chi, m)$ of $a_{i}$ being a factorobject $\left(\chi_{1}, m\right)$ of $a$ the relation

$$
\left(\chi_{1}, m\right) \leqq(\delta, d) \leqq\left(\delta_{0}, d_{0}\right)<\left(\alpha_{i}, a_{i}\right)
$$

is valid. Therefore the union of all normal factorobjects differs from $\left(\varepsilon_{a_{i}}, a_{i}\right)$ and so $a_{i}$ is indeed subdirectly irreducible. If $a_{i}$ is subdirectly irreducible, then (I) is trivially fulfilled.

REMARK. From Theorem 1 one can easily obtain BIRKHOFF's well-known theorem mentioned at the beginning of this chapter.

Dualizing Theorem 1 we obtain

THeOREM $1^{*}$. If the lattice $L_{a}^{*}$ of all normal factorobjects of an object $a \in \mathscr{C}$ is compactly generated, then $a$ is a transfree image of a free product $f=\sum_{i \in I} a_{i}\left(\varrho_{i}\right)$ by an epimorphism $\gamma$ such a way that every map $\varrho_{i} \gamma=\alpha_{i}: a_{i} \rightarrow a(i \in I)$ is a normal monomorphism. A factor $a_{i}$ of this decomposition is transfreely irreducible if and only if the following condition holds:

$\left(I^{*}\right)$ For any ideal $(m, \chi) \neq\left(a_{i}, \varepsilon_{a_{i}}\right)$ of $a_{i}$ (which is clearly a subobject $\left(m, \chi_{1}\right)$ of $\left.a\right)$ there exists an ideal $(d, \delta)$ of a such that $\left(m, \chi_{1}\right) \leqq(d, \delta)<\left(a_{i}, \alpha_{i}\right)$.

Condition $\left(\mathrm{I}^{*}\right)$ means that for the ideal $\left(a_{i}, \alpha_{i}\right)$ the object $a_{i}$ has exactly one maximal ideal $\left(d, \delta^{\prime}\right)$ (and $(d, \delta)$ is an ideal of a $\left(\delta=\delta^{\prime} \alpha_{i}\right)$ ).

To give an interpretation of Theorem $1^{*}$ we introduce the following concept. An element $k$ of a complete lattice $L$ is called a co-compact element, if $k \geqq \bigcap l_{i}$ implies $k \geqq \bigcap_{j \in J} l_{j}$ for some finite $J \subseteq I$. The lattice $L$ is said to be co-compactly generated, if $L$ is complete and every element of $L$ is an intersection of co-compact elements. Hence by Proposition 2 the condition 'the lattice $L_{a}^{*}$ of all normal factorobjects of $a$ is compactly generated' should read 'the lattice $L_{a}$ of all ideals of $a$ is co-compactly generated'. For comparison we mention that the lattice of all ideals of a ring need not be co-compactly generated and the same holds for groups too. 


\section{$\S 4$. Some applications}

In what follows $\mathscr{C}_{R}$ will denote the category of rings. As it was mentioned before, $\mathscr{C}_{R}$ satisfies axioms $\left(\mathrm{C}_{1}\right)-\left(\mathrm{C}_{10}\right)$ and condition $(\mathrm{I})$, furter the lattice $L_{A}$ of all ideals of a ring $A \in \mathscr{C}_{R}$ is compactly generated. Moreover, in $\mathscr{C}_{R}$ every map has a normal image. This means that Theorems 1 and $1^{*}$ hold for $\mathscr{C}_{R}$. On the other hand, $L_{A}$ has not to be co-compactly generated and condition $\left(I^{*}\right)$ is generally not fulfilled. However, there are some special but usual conditions which involve the validity of $\left(I^{*}\right)$ or that $L_{A}$ is co-compactly generated. Thus Theorem $1^{*}$ yields some theorems of unusual type for rings.

First of all we remark that instead of a free product of rings we speak about a free sum of rings. Further, if $A_{i}(i \in I)$ is a family of rings, then their free sum $F$ is defined as the ring $F$ consisting of all formal finite sums $\sum n_{r} \varphi_{r}$ where $n_{r}$ is an integer and $\varphi_{r}$ is a product of a finite number of elements from some $A_{i}$. For commutative rings, as it is well-known, free sum means the tensor product.

First, we show the existence of a ring the ideals of which do not form a cocompactly generated lattice.

EXAMPLE. Let $A$ be a commutative principal ideal-ring with unity and without divisors of zero. (Such a ring is e.g. the ring of rational integers.) For any ideal $J \neq 0$ of $A$ there exists an element $a \in A$ with $(a)=I$. According to RÉDEI [10], Satz 188 and 189 in $A$ there exist g.c.d. and irreducible elements. Let $p \neq 1$ be an irreducible element with $(a, p)=1$. Now $(a) \supset 0=\bigcap_{k=1}^{\infty}\left(p^{k}\right)$ is valid, but obviously $(a) \nsupseteq\left(p^{k}\right)$ for any finite $k$. Thus $(a)=I$ is not a co-compact element of the lattice $L_{A}$ of all ideals of $A$. Since $I$ was chosen arbitrarily, so $L_{A}$ is not co-compactly generated.

Now we give some sufficient conditions which guarantee that a lattice $L$ should be co-compactly generated.

Proposition 6. Every element l of a lattice $L$ is co-compact if and only if $L$ satisfies the descending chain condition. In particular, the lattice $L_{A}$ of a ring, abelian group and $R$-module A satisfying the minimum condition for ideals, subgroups and R-modules, respectively, is co-compactly generated.

Proof. Assume that each element of $L$ is co-compact, and consider a descending chain $l_{1} \supseteqq l_{2} \supseteqq l_{3} \supseteqq \ldots$ in $L$. Since also $l_{0}=\bigcap l_{n}$ is co-compact, so there exists an index $n_{0}$ with $l_{0}=l_{n_{0}}$ and the chain is finite. The inverse statement is trivial.

Proposition 7. If the ring $A$ is a discrete direct sum of rings $A_{i}(i \in I)$ with minimum condition for ideals and each $A_{i}$ has either a left or a right unity, then the ideal lattice $L_{A}$ of $A$ is co-compactly generated.

Proof. At first we prove that any ideal $B$ of $A$ is a discrete direct sum $B=\sum_{i \in I} \oplus B_{i}$ where $B_{i}$ is an ideal of $A_{i}$ for all $i \in I$. Let $b$ be an arbitrary element of $B$, then $b$ is a finite sum $b=\sum_{i \in J} a_{i}$ of elements $a_{i} \in A_{i}$. Let $e_{i}$ be, for instance, a left unity of $A_{i}$. Then we obtain $e_{i} b=a_{i} \in B \cap A_{i}$ and obviously $B_{i}=B \cap A_{i}$ is an ideal of $A$. Thus we have $B=\sum \oplus B_{i}$. Since $A_{i}$ fulfils the minimum condition 
for ideals, so there is a finite number of ideals of $A$ containing $K_{i}=B_{i}+\sum_{i \neq j \in I} \oplus A_{j}$. Therefore $K_{i}$ is a co-compact element of the lattice $L_{A}$. Further we have $B=\sum_{i \in I}^{i \neq j} \oplus B_{i}=$ $=\bigcap_{i \in I} K_{i}$ which means that $L_{A}$ is co-compactly generated.

We remark that a ring satisfying the condition of this proposition need not fulfil the minimum condition for ideals.

Let us list some types of rings which fulfil condition $\left(\mathrm{I}^{*}\right)$.

1) Every accessible subring of the ring is an ideal. A subring $S$ is called accessible in the ring $A$, if there exists a finite ascending chain of subrings $S=S_{1} \sqsubseteq$ $\subseteq S_{2} \subseteq \ldots \subseteq S_{n}=A$ where each $S_{i}$ is an ideal of $S_{i+1}(i=1,2, \ldots, n-1)$. Since any ideal of an ideal is any accessible subring, so this condition involves condition (I*) trivially. (Cf. ANDERSON-DIVINSKY-SULIŃSKI [1]).

2) Every subring of the ring is an ideal (Cf. RÉDEI [11]).

3) The ring is completely reducible, i.e. it is a discrete direct sum of simple rings. In such a ring every ideal is a direct summand. Since any ideal of a direct summand is an ideal also in the ring, so it follows condition $\left(\mathrm{I}^{*}\right)$ (Cf. JACOBSON [7] Chapter IV. 1).

4) Every subring of the ring is a direct summand (cf. F. SzÁsz [14]).

5) Every ideal of the ring is idempotent. Let $A$ be, namely, such a ring and $K$ an ideal of the ideal $I$ of $A$. By a varied form of a lemma of ANDRUNAKIEvič [2] (see also Divinsky [6], Lemma 61), we obtain

$$
\bar{K}=\bar{K}^{3} \subseteq I \cdot \bar{K} \cdot I=I(K+K A+A K+A K A) I \subseteq K \subseteq \bar{K},
$$

where $\bar{K}$ denotes the ideal of $A$ generated by the subring $K$. Thus $K$ is an ideal in $A$ too.

Important subcases of 5) are the following:

6) The ring $A$ is regular in the sense of von Neumann, i.e. for any $a \in A$ there exists an element $x \in A$ with $a=a x a$. By definition, it is clear that the ideals of such a ring are idempotent.

7) The ring $A$ is weakly regular, i.e. every right ideal of $A$ is idempotent (Cf. BROWN-MCCOY [5]).

8) The ring $A$ is biregular, i.e. every principal two-sided ideal of $A$ can be generated by a central idempotent element (Cf. ARENS-KAPLANSKY [3], BROWN-MCCOY [5] and ANDRUNAKIEVIČ [2]). If $I$ is an arbitrary ideal of the ring $A$ and $a \in I$, then there is a central idempotent element $c \in A$ such that $a \in(a)=(c)$. Hence from $c \in(c)^{2}=(a)^{2} \subseteq I^{2}$ we obtain $a \in I^{2}$ for every $a \in I$. Thus the ideals of $A$ are idempotent.

Theorem $1^{*}$ yields immediately

TheOREM 2. Let $A$ be a ring of one of the types 1)-8). If the ring $A$ is either a ring with minimum condition for ideals or a discrete direct sum of rings with left or right unity elements and the direct components satisfy the minimum condition for ideals, then there exist ideals $A_{i}(i \in I)$ of $A$ such that

(i) $A_{i}$ has exactly one maximal ideal which is an ideal also of $A$ for each $i \in I$.

(ii) every $A_{i}$ is of the same type as $A$,

(iii) $A$ is a homomorphic image of a free sum $\sum_{i \in I} B_{i}$, where $B_{i} \cong A_{i}$ holds for all $i \in I$. 
The statement that rings having one of the properties 1)-8) satisfy condition (ii), is almost trivial.

As another application, consider the category $\mathscr{C}_{G}$ of all groups. Now conditions $\left(\mathrm{C}_{1}\right)-\left(\mathrm{C}_{10}\right)$ are satisfied. Condition ( $\left.\mathrm{I}^{*}\right)$ is fulfilled, for instance, if any normal subgroup of a normal subgroup is a normal subgroup of the group, or briefly: normality is a transitive relation among the subgroups of a group. (Cf. D. S. RoBINSON [12]). From Theorem $1^{*}$ it follows immediately

Theorem 3. Let $L_{G}$ denote the lattice of all normal subgroups of a group $G$. If $L_{G}$ is co-compactly generated, and normality of subgroups of $G$ is a transitive relation, then there exist normal subgroups $G_{i}(i \in I)$ of $G$ such that

(i) each $G_{i}$ has exactly one maximal normal subgroup,

(ii) $G$ is a homomorphic image of a free product $\prod_{i \in I}^{*} F_{i}$ where $F_{i} \cong G_{i}$ holds for every $i \in I$.

Let $R$ be a ring, and consider the category $\mathscr{C}_{R}$ of all $R$-modules. $\mathscr{C}_{R}$ fulfils conditions $\left(\mathrm{C}_{1}\right)-\left(\mathrm{C}_{10}\right)$ as well as $(\mathrm{I})$ and $\left(\mathrm{I}^{*}\right)$. In $\mathscr{C}_{R}$ free sum means discrete direct sum. Hence from Theorem $1^{*}$ we obtain

THEOREM 4. If the lattice $L_{M}$ of submodules of an R-modul $M$ is co-compactly generated, then there exist submodules $M_{i}(i \in I)$ of $M$ such that $M$ is a homomorphic image of a discrete direct sum $\sum_{i \in I} \oplus N_{i}$ where $N_{i}$ is isomorphic to $M_{i}$ and $N_{i}$ has exactly one maximal submodule for each $i \in I$.

Since any abelian group can be regarded as a module over the integers, so the analogous statement to that of Theorem 4 is valid for abelian groups too.

\section{(Received 17 June 1968)}

MTA MATEMATIKAI KUTATÓ INTÉZETE, BUDAPEST, V., REÁLTANODA U. $13-15$

\section{References}

[1] R. ANDERSON-N. DivinSKY-A. SUliŃSKI, Lower radical properties for associative and alternative rings, $J$. London Math. Soc., 41, pp. 417-424.

[2] B. A. ANDRunakievič, Biregular rings (Russian), Math. Sbornik, 39 (1956), pp. 447-464.

[3] R. F. ARENS-J. Kaplansky, Topological representation of algebras, Trans. Amer. Math. Soc., 63 (1948), pp. 457-481.

[4] G. BiR KhOFF, Subdirect unions in universal algebras, Bull. Amer. Math. Soc., 50 (1944), pp. $764-768$.

[5] B. BRowN-N. H. McCoy, Some theorems on groups with application to ring theory, Trans. Amer. Math. Soc., 66 (1950), pp. 302-311.

[6] N. Divinsky, Rings and radicals (London, 1965).

[7] N. JaCOBSON, Structure of rings (Providence, 1956).

[8] A. G. Kurosch-A. Ch. Liwschitz-E. G. Schulgeifer-M. S. Zalenko, Zur Theorie der Kategorien (Berlin, 1963).

[9] B. MitcheLL, Theory of categories (New York-London, 1965).

[10] L. RÉDEI, Algebra I (Leipzig, 1959). 
[11] L. RÉDEI, Vollidealringe im weiteren Sinn. I, Acta Math. Acad. Sci. Hung., 3 (1952), pp. $243-$ 268.

[12] D. S. Robinson, Groups in which normality is a transitive relation, Proc. Cambridge Phil. Soc., 60 (1964), pp. $21-38$.

[13] A. SulińsKi, The Brown-McCoy radical in categories, Fund. Math., 59 (1966), pp. 23-41.

[14] F. SzÁsz, On rings whose every subring is a direct summand of the ring (Russian), Mat. Sbornik, 40 (1956), pp. $269-272$. 